\title{
Why the Michelson-Morley Experiment Cannot Observe the Movement of Interference Fringe
}

\author{
Tony Yuan \\ Beihang University, Beijing, China \\ Email: tony1807559167@gmail.com
}

How to cite this paper: Yuan, T. (2021) Why the Michelson-Morley Experiment Cannot Observe the Movement of Interference Fringe. Open Access Library Journal, 8: e8011.

https://doi.org/10.4236/oalib.1108011

Received: September 27, 2021

Accepted: November 7, 2021

Published: November 10, 2021

Copyright $\odot 2021$ by author(s) and Open Access Library Inc.

This work is licensed under the Creative Commons Attribution International License (CC BY 4.0).

http://creativecommons.org/licenses/by/4.0/

\begin{abstract}
The Michelson-Morley Experiment (MMX) tried to prove the existence of ether, but they did not observe the movement of interference fringes, which led to the assumption that the speed of light is constant in the inertial reference frame, which is also the theoretical basis of Einstein's special relativity (SR). So are there other possibilities that caused the experiment to fail? The Laser Interferometer Gravitational-Wave Observatory (LIGO) has observed gravitational waves, which opens up research ideas for us. If the weakest gravitational wave can raise the fluctuation of the LIGO interference fringe of light, how must we neglect the gravitational field influences on light? We raise the possibility that light is influenced by the Earth's gravitational field. In this way, MMX cannot observe the movement of interference fringe either in the air or in a vacuum environment.
\end{abstract}

\section{Subject Areas}

Classical Physics, Experimental Physics, Special Theory of Relativity

\section{Keywords}

Michelson-Morley Experiment, Gravitational Wave, Gravitational Field, LIGO

\section{Introduction}

This paper provides a detailed analysis in several parts: the influence of water on the speed of a car, the influence of air on light propagation, the influence of gravitational field on light propagation, Eddington Observation, mass-energy equation, fiber optic gyroscope and explanation of the Sagnac effect. 


\section{Influence of Water Flow on Car Speed}

Consider a car in motion on a water-covered road as shown in Figure 1. Does it signify that surface water is necessary for car driving? I believe that everyone will answer "no", for a car can still drive smoothly without surface water on the road. Indeed, driving does not need water as a medium. On the contrary, water can affect a car's driving speed, which is assumed $v_{\text {water }}$. When $v_{\text {water }}=0$, water is static against the ground, and the driving speed of the car against the ground is $v_{c a r}$. Then, the driving speed of the car parallel to the flow direction is

$$
v_{\text {car }}+v_{\text {water }} \text {, }
$$

and the driving speed of car in reverse against the flow direction is

$$
v_{\text {car }}-v_{\text {water }} \text {. }
$$

Water flow affects the car's speed, as the car is seized by water.

If the water flow direction is not in parallel with that of the car, we only need to decompose flow velocity into its component $v_{x}$ parallel to the car direction and its vertical direction $v_{y}$. The logic is the same. For simplicity, assume that the car's velocity is in parallel with the water flow.

There are two buoys, $\mathrm{A}$ and $\mathrm{B}$, on the water surface as shown in Figure 2; the distance between them is $\mathrm{L}$. They are static against the water surface. The direction of water flow is from A to B. Now, the car needs to drive from $A$ to $B$, then from B to A. From this, we need to measure the duration $T$.

First, check the duration from A to B. The car drives parallel to the water, with the speed being $v_{c a r}+v_{\text {water }}$. As the buoy is static against the water surface, it also moves along the same direction with the water flow velocity. Therefore, the duration is

$$
T=L /\left(v_{\text {car }}+v_{\text {water }}-v_{\text {water }}\right)=L / v_{\text {car }} .
$$

Then, considering $\mathrm{B}$ to $\mathrm{A}$, the car drives against the water, with its speed being $v_{c a r}-v_{\text {water }}$. As the buoy is static against the water surface, it also moves in the reverse direction with the water flow velocity. Therefore, the duration is

$$
T=L /\left(v_{\text {car }}-v_{\text {water }}+v_{\text {water }}\right)=L / v_{\text {car }} .
$$

The time required for the car to go from A to B and from B to A is the same. Such a duration has nothing to do with $v_{\text {water }}$. This conclusion is likewise valid

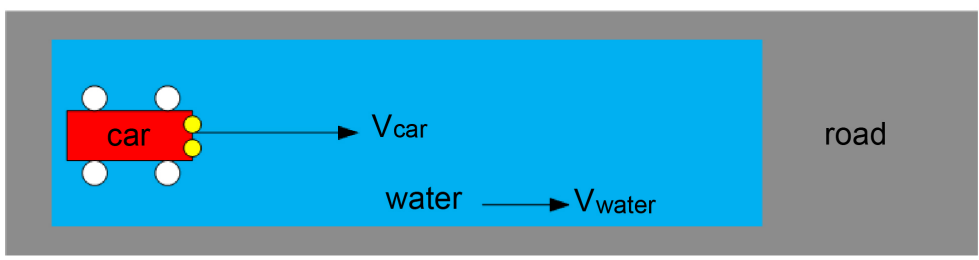

Figure 1. Influence of water flow on car speed.

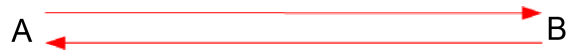

Figure 2. A to B to A. 
even if the water flow direction is not parallel with that of the car. Taking the water as the reference object to measure the car's speed, the speed is

$$
L / T=L /\left(L / v_{c a r}\right)=v_{c a r} .
$$

Considerably, those people in the car driving at a constant velocity can measure a toy car's speed, which remains the same no matter what the driving direction is. However, when taking the ground as the reference object, the speed of the toy car varies with the movement direction. In this case, speed is different when the reference system is different.

\section{Influence of Air on Light Transmission}

The above analysis transpires for the sake of light analysis. A beam of light transmits in the air. Does it signify that air is necessary to transmit light? The answer is no; light transmission does not need air as a medium.

Similarly, the existence of air can influence the speed of light transmission. The speed of light moving parallel to the air is faster than that of light moving against the air. In this case, light is seized by air. Comparing the above scenarios:

car......counterpart......light

water......counterpart.......air

road......counterpart......space

water moves against road......counterpart.......air moves against space

Now, let us focus on light. Assume that the speed of light in a vacuum is $c$, and the air and the ground are relatively static. To simplify, set the direction of light and the Earth's revolution velocity as parallel. In a short time, we can regard the Earth's motion as a uniformly linear motion.

Place two signs corresponding to A and B on the ground. The direction from $A$ to $B$ is in line with the direction of the Earth's revolution. When the ground is static against point $\mathrm{O}$ in space, the speed of light in static air is $C_{0}$. Thus, when the light moves from $\mathrm{A}$ to $\mathrm{B}$, the required duration is

$$
L /\left(c_{0}+v_{\text {earth }}-v_{\text {earth }}\right)=L / c_{0} .
$$

When the light moves from $\mathrm{B}$ to $\mathrm{A}$, the required duration is

$$
L /\left(c_{0}-v_{\text {earth }}+v_{\text {earth }}\right)=L / c_{0} .
$$

Therefore, irrespective of the speed of the ground against point $\mathrm{O}$ in the space, it does not impact the duration of light moving A to B or from B to A. Similarly, if the water surface is the reference object, the car's speed does not change irrespective of the driving direction; the light takes the Earth as the reference, and its speed does not change. Can we say that the existence of air is the fundamental reason for the phenomenon that MMX [1] cannot observe the movement of interference fringe of light? No!

\section{Influence of Gravitational Field on Light Propagation}

The LIGO [2] interferometer of the United States improves the MMX. It places 
the experiment entirely in a vacuum environment, wherein light is not affected by air anymore. If the cause for the failure of the MMX lies in the air, LIGO should observe the movement of fringe, but it does not. Following our above deduction, the duration of light moving from $\mathrm{A}$ to $\mathrm{B}$ should be

$$
L /\left(c-v_{\text {earth }}\right)
$$

and that from B to A should be

$$
L /\left(c+v_{\text {earth }}\right)
$$

By classical physics, it shows that the required duration varies with $v_{\text {earth }}$ as shown in Figure 3. Then, it should be easy to find the fluctuation of fringe, but we cannot. Does it further demonstrate the theory of Einstein, in which light speed is constant no matter the inertial reference system is? Or are there other possibilities? The greatest finding of LIGO is the discovery of gravitational waves [3] [4]. The weakest gravitational wave raises the fluctuation of interference fringe; that is, the gravitational wave affects light speed and leads to LIGO optical path difference changes in horizontal and vertical directions. Such a finding is significant, which demonstrates that gravitational waves can impact light transmission.

If the weakest gravitational wave can raise the fluctuation of the LIGO interference fringe of light, how should we neglect gravitational field [5] influences on light? We raise the possibility that light is influenced by both the gravitational field and the air in the Earth. In this way, the MMX cannot find the movement of interference fringe either in the air or in a vacuum environment. The water flow seizes the car; when the water surface serves as the reference object, the car's speed is constant. Similarly, the gravitational field seizes light; when the ground serves as the reference object, the speed of light is constant. Additionally, the car speed varies, thus adding the speed of water flow.

Is the light speed viable when point $\mathrm{O}$ in space serves as the reference object? Can the movement speed of the Earth's gravitational field be added?

\section{Eddington Observation}

Such an observation demonstrates the correctness of general relativity and proves

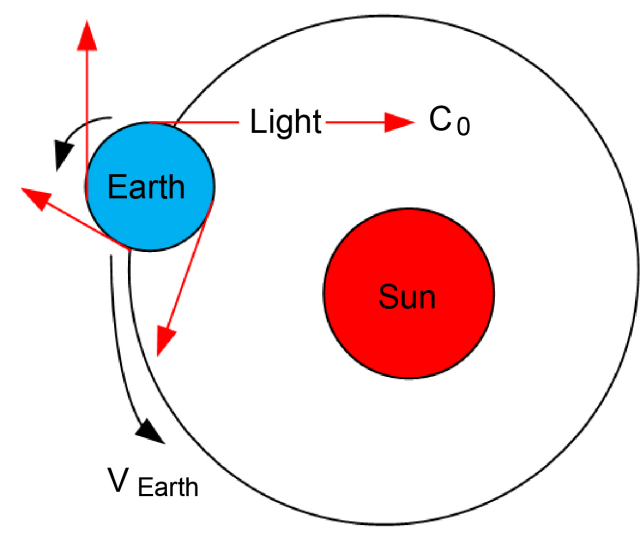

Figure 3. Light propagation model on the earth. 
that light's speed is influenced by the gravitational field. Under this influence, the velocity component of light decreases in the original direction, while increasing the gravity's direction. In this case, we cannot measure whether there is any change of $c^{\prime}$ near a fixed star, but determine only that the light's direction changes as shown in Figure 4. However, as light moves away from a fixed star, the measured speed of light is still $c$. Is it like the sound wave in the air? The speed of sound in the plane adds to the plane's speed. However, as sound travels out of the plane window, its speed returns as airspeed, which is static against the ground. Hence, they are similar to each other, with the difference between them is that light is pulled by the gravitational field, while the aircraft pulls the sound waves because of the medium (air inside the aircraft).

Is the speed of light definitely constant? Can the MMX lead to the conclusion that light speed is constant under any inertial reference system?

Two reasons can lead to the bending of an object's moving path as shown in Figure 5. One is the effect of force, resulting in speed in a vertical direction; the other is refraction caused by an inhomogeneous medium or field, resulting in bending of the path. In this case, the bending of light is probably caused by the latter. A gravitational field surrounds the sun; as the gravitational field goes in, it becomes stronger. When light passes through the sun's gravitational field, it bends like refraction due to the gravitational field's inhomogeneity. The bending of this path is different from bending caused by the direct action of gravity. Therefore, it is not suitable to calculate the bending angle of light using the classical theory of flat throw.

This paper analyzes that, whether the sun moves in the same direction, in the opposite direction, or at a certain angle, the speed of light relative to the sun does not change. In the calculation of the theory of flat throw, since there is an acceleration process under the gravity's pull at the beginning before the acceleration caused by gravity becomes negative, the light becomes farther away from the sun and is less affected by gravity, making the deflection angle of light smaller. Thus, the deflection angle of light calculated by the classical theory of flat throw is too small. There is nothing wrong with Newtonian mechanics, but it

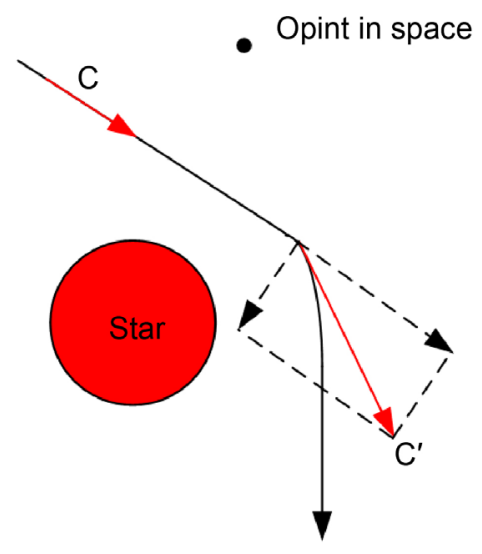

Figure 4. Bending of light. 


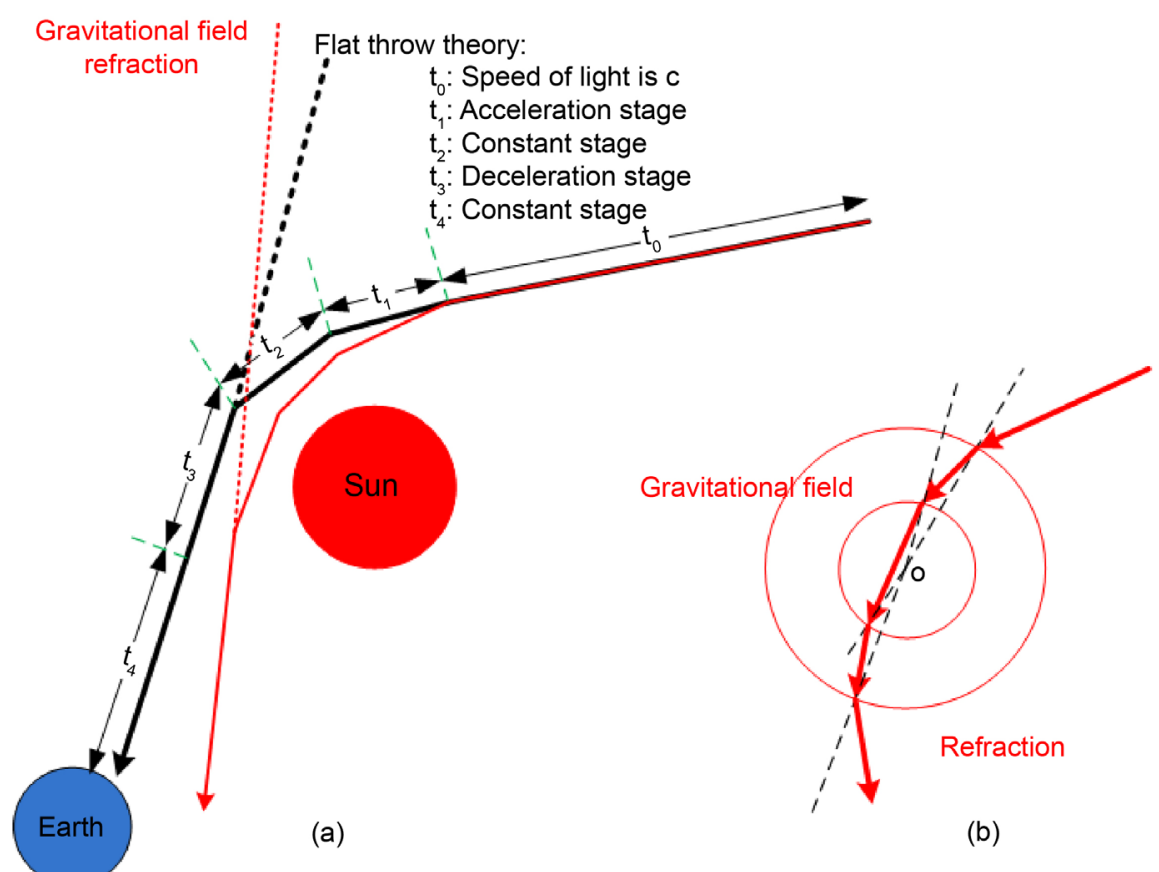

Figure 5. Eddington observation.

was not used accurately at that time.

\section{The Mass Energy Equation}

$$
E=m * c * c
$$

The above equation does not need relativity as the basis. Hence, we use classical Newtonian mechanics to derive the mass-energy equation.

1) Presumably, it takes time $t_{0}$ for a substance with mass $m$ to accelerate from $v=0$ to $v=c$. Then, it is assumed that this process entails uniform acceleration, as shown, in Figure 6.

Therefore, we can obtain the displacement:

$$
S=c * t_{0} / 2
$$

The work necessarily done is:

$$
W=F * S=m * a * S=m * a * c * t_{0} / 2=m * c * a * t_{0} / 2=m * c * c / 2
$$

2) However, it is definitely not accelerating evenly, as shown, in Figure 7:

3) Upon approximation, displacement can be $s=c * t_{0}$.

4) Acceleration is nonlinear as a function of time $t$. Its cumulative effect on time is the final speed $c$.

$$
W=\int_{0}^{t_{0}} F(t) * c * \mathrm{~d} t=\int_{0}^{t_{0}} m * a(t) * c * \mathrm{~d} t=m * c * \int_{0}^{t_{0}} a(t) * \mathrm{~d} t=m * c * c
$$

Science is rigorous, basing on the logical analysis and mathematical derivation of scientific experiments. Hence, we use classical Newtonian physics to analyze the MMX and derive the mass-energy equation. We need not assume that the speed of light is constant. 


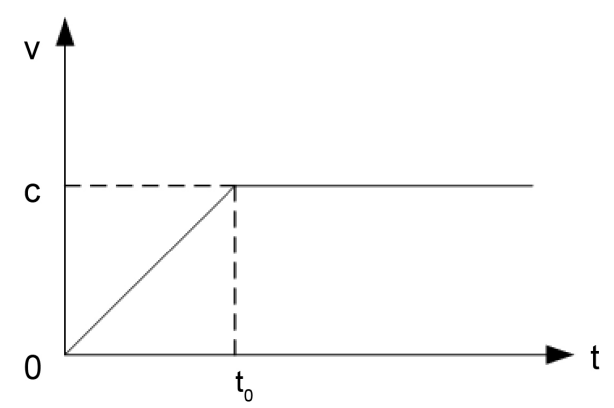

Figure 6. Uniform acceleration linear motion.

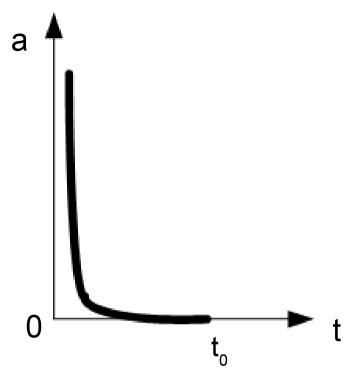

(a)

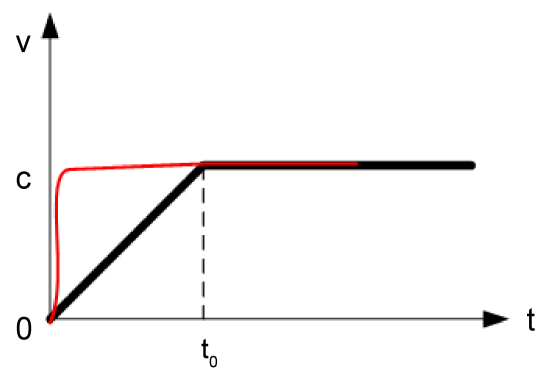

(b)

Figure 7. Linear motion with massive acceleration.

\section{Explanation of Sagnac Effect}

Scholars have extensively used the fiber optic gyroscope, as classical Newtonian mechanics can explain its working principle well. In determining the speed of the fiber optic gyroscope relative to the ring, we assume that the speed of light in the ring is $c_{0}$ when the fiber optic ring is stationary.

The radius of the ring is $R$, while its angular rate is $w$ as shown in Figure 8 .

Speed of light along the ring:

$$
v_{1}=c_{0}-w * R
$$

Speed of light against the ring:

$$
v_{2}=c_{0}+w * R
$$

Therefore, the time difference when the light is emitted from the two directions meets:

$$
\delta t=2 \pi * R / v_{1}-2 \pi * R / v_{2},
$$

substitute $v_{1}, v_{2}$, for finishing, $\delta t$ is about equal to:

$$
4 \pi * R * R * w /\left(c_{0} * c_{0}\right) .
$$

The optical path difference is

$$
\delta S=c_{0} * \delta t=4 \pi * R * R * w / c_{0} .
$$

The number of corresponding fringe movement is:

$$
\text { num }=\delta S / \text { wavelength }=4 \pi * R * R * w /\left(c_{0} * \text { wavelength }\right) .
$$




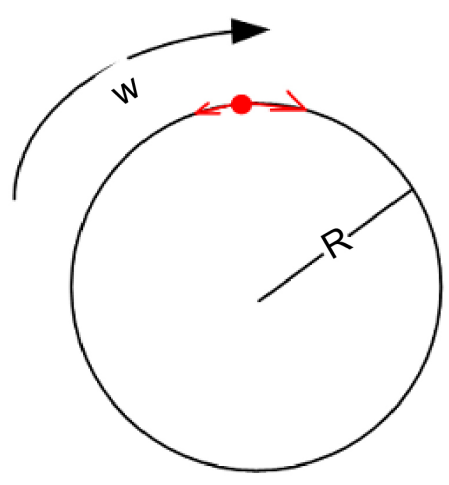

Figure 8. Explanation of Sagnac effect.

By the number of moving stripes, we can calculate the rate of $w$. For instance, assuming that the number of moving stripes is $1, R=1 \mathrm{~m}$, and the wavelength of light is $500 \mathrm{~nm}$, then

$$
w=n u m * c_{0} * \text { wavelength } /(4 \pi * R * R)=11.9(\text { radians } / \mathrm{s}) .
$$

The calculated results are in good agreement with the measured results. Nevertheless, when looking at formulas (14) and (15), they are directed against the constant speed of light. Is it a coincidence that they are wrong? Due to the extensive use of the fiber optic gyroscope, it is sufficient to depict its correctness.

Can the Earth's rotation be measured when the fiber optic gyroscope is stationary relative to the ground? Our previous analysis makes it impossible to measure Earth's rotation because a gravitational field holds the light. Engineers who produce fiber optic gyroscopes have also confirmed this finding.

We can do an experiment, put fiber optic gyroscope on the north pole of the moon and let it rotate at the speed of the Earth's rotation, you will find that the speed is no longer displayed as 0 , but the speed of Earth's minus the speed of the Moon's. And if you let fiber optic gyroscope stand still relative to the lunar ground, then you will find that the speed is displayed as 0 .

The light is affected by the gravitational field.

\section{Conclusions}

Scientists were unable to provide a reasonable explanation for the MMX at the time; hence, the assumption existed that the speed of light is constant. Einstein established special relativity based on this. Nevertheless, LIGO provides a direction for thinking and applying classical physics theories, from which we can explain various scientific phenomena well, including the MMX. Indeed, some physical phenomena can no longer be explained by the constant speed of light, including GR, which denies a constant speed of light.

Light is affected by the gravitational field. In this way, the MMX cannot find the movement of interference fringe either in the air or in a vacuum environment. 


\section{Conflicts of Interest}

The author declares no conflicts of interest.

\section{References}

[1] Shankland, R.S. (1964) Michelson-Morley Experiment. American Journal of Physics, 31, 16-35. https://doi.org/10.1119/1.1970063

[2] LIGO: The Search for Gravitational Waves. National Science Foundation. https://www.nsf.gov/

[3] Barish, B.C. (1996) The Detection of Gravitational Waves. Caltech.

[4] Gertsenshtein, M.E. and Pustovoit, V.I. (1962) On the Detection of Low Frequency Gravitational Waves. JETP, 43, 605-607.

[5] Physics: Fundamental Forces and the Synthesis of Theory-Encyclopedia.com. 